OPEN ACCESS

Edited by:

Yuji Morita,

Meiji Pharmaceutical University, Japan

Reviewed by: Kenneth Milan Peterson, Louisiana State University Health Sciences Center, United States Jeffrey H. Withey, Wayne State University, United States

*Correspondence: Yiquan Zhang zhangyiquanq@163.com Biao Kan kanbiao@icdc.cn

Specialty section: This article was submitted to Infectious Diseases,

a section of the journal Frontiers in Microbiology

Received: 16 January 2020 Accepted: 26 March 2020 Published: 17 April 2020

Citation:

Gao H, Zhang J, Lou J, Li J, Qin $Q$, Shi $Q$, Zhang Y and Kan $B$ (2020) Direct Binding and Regulation by Fur and HapR of the Intermediate Regulator and Virulence Factor Genes Within the ToxR Virulence Regulon in Vibrio cholerae.

Front. Microbiol. 11:709. doi: 10.3389/fmicb.2020.00709

\section{Direct Binding and Regulation by Fur and HapR of the Intermediate Regulator and Virulence Factor Genes Within the ToxR Virulence Regulon in Vibrio cholerae}

\author{
He Gao ${ }^{1}$, Jingyun Zhang ${ }^{1}$, Jing Lou ${ }^{1}$, Jie Li ${ }^{1}$, Qin Qin ${ }^{1}$, Qiannan Shi ${ }^{1}$, Yiquan Zhang ${ }^{2 *}$ and \\ Biao Kan ${ }^{1 *}$
}

\footnotetext{
' State Key Laboratory for Infectious Disease Prevention and Control, National Institute for Communicable Disease Control and Prevention, Chinese Center for Disease Control and Prevention, Beijing, China, ${ }^{2}$ School of Medicine, Jiangsu University, Zhenjiang, China
}

Cholera toxin (CT) and toxin coregulated pilus (TCP, TcpA is the major subunit) are two major virulence factors of Vibrio cholerae, both of which play critical roles in developing severe diarrhea in human. Expression of CT and TCP is under the tight control of the regulatory cascade known as the ToxR virulence regulon, which is composed of three regulators ToxR, TcpP, and ToxT. Besides, their expression is also regulated by the quorum sensing (QS) master regulator HapR and the regulatory protein Fur. Though transcription of $t c p P$, toxT, and/or tcpA are reported to be regulated by HapR and Fur, to date there are no studies to verify their direct regulations. In the present study, we showed that HapR directly repress the transcription of $t c p P$ and $t c p A$ by binding to their promoter regions, and possibly repress tox $T$ transcription in an indirect manner. Fur directly activated the transcription of tcp $P$, toxT, and tcpA by binding to their promoters. Taking account of the sequential expression of hapR, fur, tcp $P$, toxT, and tcp $A$ in the different growth phases of $V$. cholerae, we deduce that at the early midlogarithmic growth phase, Fur binds to the promoters of tcpP, toxT, and tcpA to activate their transcription; while at the later mid-logarithmic growth phase, HapR can bind to the promoters of $t c p P$ and $t c p A$ to repress their transcription. Our study reveals the new recognition in the virulence regulatory pathways in $V$. cholerae and suggests the complicated and subtle regulation network with the growth density dependence.

Keywords: Vibrio cholerae, toxin coregulated pilus, cholera toxin, HapR, Fur

\section{INTRODUCTION}

Vibrio cholerae is a Gram-negative bacterium that naturally inhabits salty coastal waters and estuaries (Clemens et al., 2017), and some are the causative agent of cholera. Two virulence factors, cholera toxin (CT) and toxin coregulated pilus (TCP), are considered the most closely connected to cholera. CT, encoded by the $\operatorname{ctx} A B$ operon in the $V$. cholerae lysogenic phage CTX $\Phi$, is an $\mathrm{AB}_{5}$ toxin that consists of a single catalytic A-subunit and a pentamer of B-subunits 
(Merritt and Hol, 1995). It can enhance the concentration of intracellular cyclic AMP, which then causes an imbalance in electrolyte transport across the intestinal epithelial cell membrane, resulting in the secretion of water and electrolytes into the bowel accompanied by severe watery diarrhea, which may lead to death without timely treatment (Clemens et al., 2017). TCP, the subunit of which is encoded by $t c p A$ (in the tcpABQCRDSTEF operon), is essential for colonization of $V$. cholerae in the small intestine at the early stage of infection (Kirn et al., 2000). It also functions as the receptor for the CTXФ (Waldor and Mekalanos, 1996).

The expression of TCP and CT is tightly regulated by a regulatory cascade, referred to as the ToxR virulence regulon (Childers and Klose, 2007). Under virulence inducing growth conditions, ToxR cooperates with TcpP to bind to the promoter region of toxT to activate its transcription, and ToxT, in turn, activates the transcription of $\operatorname{ctx} A B$ and $\operatorname{tcp} A$ (DiRita et al., 1991; Krukonis et al., 2000; Goss et al., 2013). ToxR alone also can directly activate $c t x A B$ transcription in the presence of bile acids (Hung and Mekalanos, 2005). While under non-inducing growth conditions, TcpP and ToxT are proteolytically degraded in order to terminate virulence gene expression (Matson and DiRita, 2005; Abuaita and Withey, 2011). The genes for TCP and CT production are also regulated by quorum sensing (QS) (Miller et al., 2002; Zhu et al., 2002), a cell-to-cell communication process that bacteria use to monitor their cell density by detecting the extracellular concentration of autoinducers (AIs), the signaling molecules (Ball et al., 2017). In vibrios, AphA and LuxR orthologs (referred to as the HCD master regulators, HMRs) represent the terminal master regulator of QS operating at low cell density (LCD) and high cell density (HCD), respectively (Lu et al., 2018). AphA, which has interaction with AphB, binds to the promoter of tcpPH to activate its transcription (Kovacikova et al., 2004). HapR (the homologous protein of LuxR) represses the transcription of $t c p P H$ via binding and repression of $a p h A$ transcription (Kovacikova and Skorupski, 2002). The global regulator cAMPCRP represses $t c p P H$ transcription via its ability to influence AphA- and AphB-dependent transcriptional activation of $t c p P H$. This is because the cAMP-CRP binding site is completely within the binding sites of AphA and AphB (Kovacikova and Skorupski, 2001). H-NS also has roles in silencing the expression of TCP and $\mathrm{CT}$ by binding and repression of $c t x, t c p$, and tox $T$ promoters (Nye et al., 2000; Stonehouse et al., 2011). In addition, the ferric uptake regulator Fur seems to have positive regulatory activity on TCP production, because deletion of fur repressed tcp transcription and exhibited very weak autoagglutination, one indicator of the capacity of $V$. cholerae infection in vivo (Mey et al., 2005).

Although HapR repression of TCP and CT via repression of AphA has been demonstrated, whether HapR can directly regulate the genes within the ToxR virulence regulon or not, needs to be further investigated. In addition, the mechanisms of the Fur-dependent activation of TCP expression are also unclear. Moreover, transcription of fur was under the direct control of HapR, and HapR coordinates with Fur to regulate hly $A$ transcription (Gao et al., 2018), suggesting Fur integrated into QS to co-regulate gene expression in $V$. cholerae.

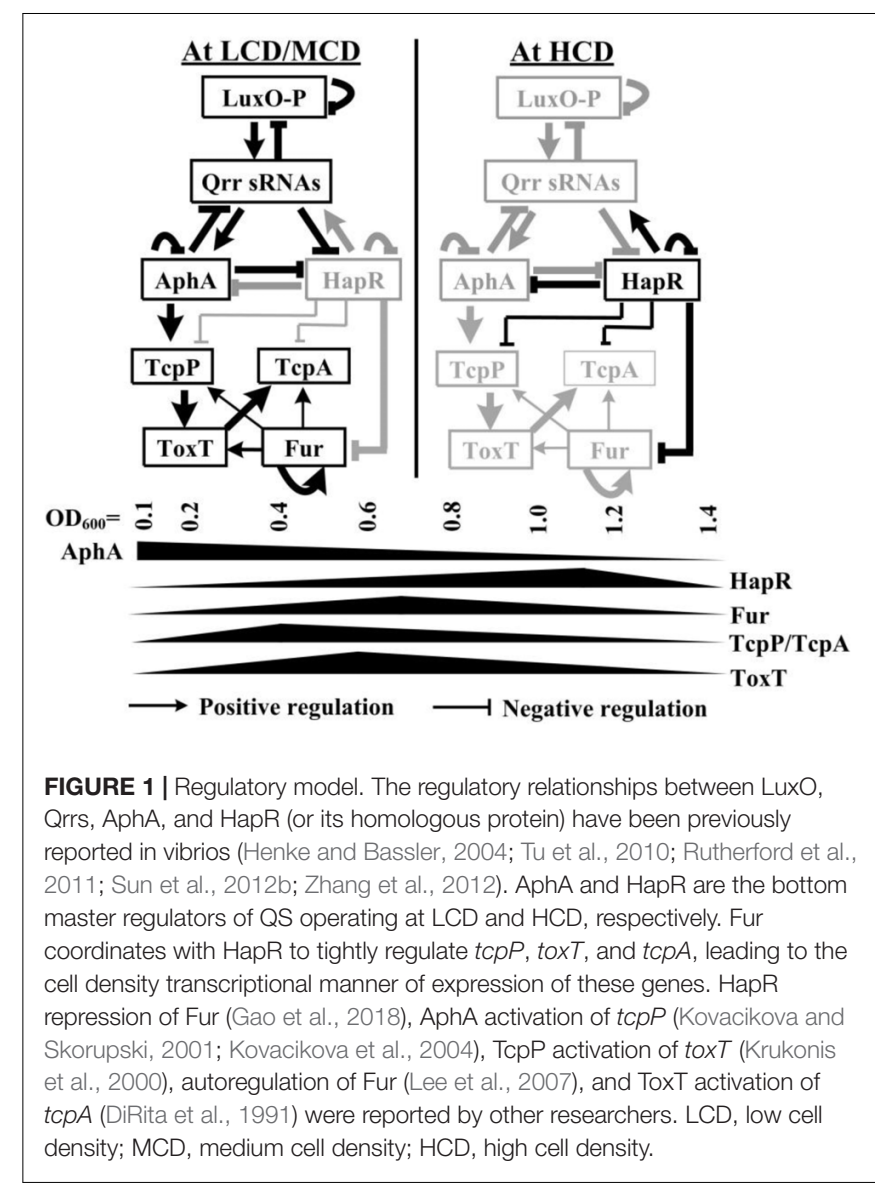

In the present study, we showed that transcription of tcpP, toxT, and tcpA were all cell-density dependent, which may be due to the coordinated regulation of Fur and HapR (Figure 1). At the $\mathrm{OD}_{600}$ value of about 0.7 , Fur binds to the promoters of $t c p P$, toxT, and $t c p A$ to activate their transcription; while at the $\mathrm{OD}_{600}$ value of about 1.0, the QS regulator HapR directly represses the transcription of tcp $P$ and $t c p A$, but it indirectly represses tox $T$ transcription. The data enriched the regulatory networks that control the expression of virulence determinants in $V$. cholerae, which promotes a deeper understanding of the pathogenic mechanisms of the pathogen.

\section{MATERIALS AND METHODS}

\section{Bacterial Strains and Cultural Conditions}

Vibrio cholerae El Tor serogroup O1 strain C7258 (Peru, 1991) was used as the derivative (wild type, WT). Non-polar fur and $h a p R$ single-gene deletion mutants $\Delta$ fur and $\Delta$ hapR derived from the WT strain were constructed in our previous study (Gao et al., 2018). The deletion mutant of lacZ (designated as $\Delta$ lacZ) was constructed from WT by homologous recombination using the suicide plasmid pWM91, which was similarly performed as previously described (Wu et al., 2015). All primers used in the present work are listed in Table $\mathbf{1 .}$ 
TABLE 1 | Oligonucleotide primers used in this study.

\begin{tabular}{|c|c|}
\hline Target & Primers (forward/reverse, $5^{\prime}-3^{\prime}$ ) \\
\hline \multicolumn{2}{|c|}{ Construction of mutants } \\
\hline \multirow[t]{3}{*}{ fur } & CGGGATCCTTCGTGTAAGGCAGCAGTAATC/CAGAGCGTAAAGCCTATGGATACTाTCCTGTTGATGTTC \\
\hline & GAACATCAACAGGAAAGTATCCATAGGCTITACGCTCTG/GGACTAGTAGATGAAGATGGTGTGGGAAAC \\
\hline & CGGGATCCTTCGTGTAAGGCAGCAGTAATC/GGACTAGTAGATGAAGATGGTGTGGGAAAC \\
\hline \multirow[t]{3}{*}{ hapR } & GCGGGATCCCCAGCAATACATCTITACC/GTGCTGCCCAAGAAAAGGGGTATATCCTTGCC \\
\hline & GGCAAGGATATACCCCПTाCTTGGGCAGCAC/GCGACTAGTAACTCACCAAAACCTTC \\
\hline & GCGGGATCCCCAGCAATACATCTITACC/GCGACTAGTAACTCACCAAAACCTTC \\
\hline \multirow[t]{3}{*}{ lacZ } & GCGGGATCCCACGGAGGGAAGGGTAAA/CCTTAAGGCTCTCTGGCCCCTCAAGCCGAGGAGTAAAG \\
\hline & CПTACTCCTCGGCTTGAGGGGCCAGAGAGCCTTAAGG/GGACTAGTCAGCCCAGACAGTGAAGG \\
\hline & GCGGGATCCCACGGAGGGAAGGGTAAA/GGACTAGTCAGCCCAGACAGTGAAGG \\
\hline \multicolumn{2}{|c|}{ Protein expression } \\
\hline fur & GCGGGATCCATGTCAGACAATAACCAAG/GCGAAGCTITATTCTTCGGCTTGTGAG \\
\hline hapR & GCGGGATCCATGGACGCATCAATCGAAAAAC/GCGAAGCTTCTAGTTCTTATAGATACACAG \\
\hline \multicolumn{2}{|l|}{ qPCR } \\
\hline tcpP & GCACAAGATCCAATGAAGCC/CTGGTTCTITGATTGCCTGAG \\
\hline tcpA & TGGTCTCAGCGGGTGTTG/CATITGCGTITGCGGTAGC \\
\hline toxT & TाTCAGGGTTCTTCTCG/ACAAATATCTGCCCAACG \\
\hline toxR & गाTाTGGCGAGAGCAAGG/TCTTCTTCAACCGTाTCCACTC \\
\hline recA & AAGATTGGTGTGATGTTGGTA/CACTTCTTCGCCTTCTTGA \\
\hline \multicolumn{2}{|c|}{ Primer extension } \\
\hline tcpA & /CCAGAACAATGATTACTTC-HEX \\
\hline \multicolumn{2}{|c|}{ Luminescence assay } \\
\hline tcpP & GCGGAGCTCGTGCCTGCTGAGAACTAA/GCGGGATCCCAAAGGTTATCGGGAAAT \\
\hline tcpA & GCGGAGCTCTCCCGACTACTCAGAAAG/GCGGGATCCATITATATAACTCCACC \\
\hline toxT & GCGGAGCTCGTGAATGTTGGTGG/GCGGGATCCTGCGTTCTACTCTG \\
\hline toxR & GCGGAGCTCTCCGCACCGTCACCGC/GCGGGATCCCTAATGTCCCAGTATC \\
\hline fur & GCGGAGCTCGCATCAAGGCATAAACGG/GCGACTAGTATACTTCCTGTTGATGTTC \\
\hline hapR & GCGGAGCTCCCAGCAATACATCTITACC/GCGACTAGTTGAGGCGATAGCCGAGTT \\
\hline \multicolumn{2}{|c|}{ DNase I footprinting } \\
\hline \multirow[t]{2}{*}{ tcpP } & GTAAAACGACGGCCAGTCAGGAAAGATAATGTAACC/CAGGAAACAGCTATGACGTGTACCAATCAGCCTा \\
\hline & GTAAAACGACGGCCAGTGTGCCTGCTGAGAACTAA/CAGGAAACAGCTATGACGGGCTाIITAACTाG \\
\hline tcpA & GTAAAACGACGGCCAGTTCCCAATTGGTTGGCTC/CAGGAAACAGCTATGACCATATTTATATAACTCCACC \\
\hline \multirow[t]{2}{*}{ toxt } & GTAAAACGACGGCCAGTCAGGTCGATITCTAC/CAGGAAACAGCTATGACTTCCACTATCTATCC \\
\hline & GTAAAACGACGGCCAGTCAGGTCGATITCTTAC/CAGGAAACAGCTATGACCCTTAAACTGCACATC \\
\hline toxR & GTAAAACGACGGCCAGTTCCGCACCGTCACCGC/CAGGAAACAGCTATGACCCAATATGACTCATCG \\
\hline M13 & FAM-GTAAAACGACGGCCAGT/CAGGAAACAGCTATGAC-HEX \\
\hline
\end{tabular}

All strains were maintained at $-80^{\circ} \mathrm{C}$ in $\mathrm{LB}$ broth $[1 \%$ tryptone (Oxoid), $0.5 \%$ yeast extract (Oxoid), and $1 \% \mathrm{NaCl}$ (Merck Millipore)] containing 30\% glycerol (v/v). Unless stated otherwise, $V$. cholerae strains were cultured with AKI [1.5\% Bacto peptone (BD Biosciences), 0.4\% yeast extract (Oxoid), $0.5 \% \mathrm{NaCl}$ (Merck Millipore), and $0.3 \% \mathrm{NaHCO}_{3}$ (Merck Millipore)] of the TCP-induced conditions as previously described (Iwanaga et al., 1986). When appropriate, the culture medium was supplemented with $100 \mu \mathrm{g} / \mathrm{ml}$ ampicillin or $50 \mu \mathrm{g} / \mathrm{ml}$ kanamycine.

\section{Competition Assay}

In vivo competition assay was performed as previously described (Tamayo et al., 2010). The $\Delta l a c Z, \Delta$ fur and $\Delta$ hapR strains were grown overnight on LB agar containing the appropriate antibiotics at $37^{\circ} \mathrm{C}$. For each strain approximately 10 colonies were resuspended in $1 \mathrm{ml}$ phosphate-buffered saline (PBS).
The strains were mixed 1:1 and adjusted to a final concentration of approximately $10^{6} \mathrm{CFU} / \mathrm{ml}$. Five-day-old CD-1 infant mice were used as the infection model, and each mouse was intragastrically inoculated with $100 \mu \mathrm{l}$ (about $10^{5} \mathrm{CFU}$ ) of the mixture (Tamayo et al., 2010). Mice were sacrificed at $20 \mathrm{~h}$ post-inoculation, and the bacterial cells in the intestines were enumerated on LB agar plates containing $40 \mu \mathrm{g} / \mathrm{ml}$ of 5-bromo-4-chloro-3-indolyl- $\beta$-D-galactopyranoside ( $X$-gal) to differentiate the $\triangle l a c Z$ and the regulatory gene mutant colonies and to determine the input ratios and bacterial titers. In vitro competitions were performed in parallel to calculate the input ratios by inoculating $2 \mu \mathrm{l}$ of the mix into $1 \mathrm{ml} \mathrm{LB}$ and incubating overnight at $37^{\circ} \mathrm{C}$ with aeration. The competition index (CI) of the input and the output were calculated as the blue/white ratio of the mutant/ $\triangle$ lacZ.

This work was performed in strict accordance with animal protocols approved by the ethics committee of the 
National Institute for Communicable Disease Control and Prevention, China CDC.

\section{Quantitative PCR (qPCR)}

Extraction of bacterial total RNAs, generation of cDNAs, and operation of qPCR were performed as previously described (Gao et al., 2011). The relative mRNA levels were determined based on the standard curve of recA (reference gene) expression for each RNA preparation. The qPCR assay was performed with at least three independent biological samples.

\section{Luminescence Assay}

For the luminescence assay (Xu et al., 2010; Gao et al., 2018), the promoter DNA region of each target gene was cloned into the pBBRlux harboring a promoterless $l u x C D A B E$ reporter gene and a chloramphenicol resistance gene. The recombinant plasmid was transferred into WT and each mutant strain, respectively. Strains transformed with recombinant plasmids were cultivated in AKI-inducing conditions and harvested at the required cell densities. The luminescence was measured using an Infinite ${ }^{\circledR}$ 200 Pro NanoQuant (Tecan, Switzerland). The lux activity was calculated as light units/OD 600 .

\section{DNase I Footprinting Assay}

The recombinant proteins His-Fur and His-HapR were expressed using the pET28a plasmid and the Escherichia coli BL21 $2 \mathrm{DE} 3$ cells (Kleber-Janke and Becker, 2000; Gao et al., 2008). The purified recombinant proteins were concentrated to a final concentration of 0.3 to $0.6 \mathrm{mg} / \mathrm{ml}$. DNase I footprinting and sequencing assays were carried out as previously described (Gao et al., 2017; Zhang et al., 2017b). Briefly, the FAM (or HEX)labeled DNA probes were incubated with the increasing amounts of His-tagged protein, and digested by the optimized RQ1 RNaseFree DNase I (Promega), and then analyzed using an ABI 3500XL DNA Genetic analyzer with GeneMarker software 2.2, while the DNA sequencing products were surveyed with Sequence Scanner software v1.0.

\section{Primer Extension Assay}

The primer extension assay was essentially performed as previously described (Gao et al., 2018). Twelve micrograms of total RNA was annealed with 1 pmol of $5^{\prime}$ - HEX-labeled reverse primer to generate cDNAs using the Primer Extension System (Promega), and the products of primer extension were then analyzed using the ABI 3500XL DNA Genetic analyzer.

\section{Experimental Replicates and Statistical Methods}

The competition assay was done at least three independent times with similar results. The data of DNase I footprinting and primer extension were done at least two independent times. The luminescence assay and qPCR were performed with at least three independent bacterial cultures, and the values were expressed as the mean \pm standard deviation (SD). Paired Student's $t$-test was used to calculate significant differences, $P<0.01$ was considered to indicate statistical significance.

\section{RESULTS}

\section{Binding Sites of HapR/Fur Were Predicted Within the Regulatory Regions of $\operatorname{tcp} P, \operatorname{tcp} A$, and toxT}

Toxin coregulated pilus production is under the control of a tightly regulated signaling cascade composed of ToxR, TcpP/H, and ToxT (Childers and Klose, 2007). It has been reported that HapR represses tcpA transcription through binding and repression of aphA (Kovacikova and Skorupski, 2002), however, it remains unknown whether HapR can directly repress $t c p A$ or not. Here, the 700 bp upstream regions of toxR, tcpP, toxT, and $t c p A$ were retrieved from the genome sequence of strain C7258, and the DNA binding box of the HMRs (TATTGATAAATTTATCAATA) in vibrios (Zhang et al., 2012) was used to statistically predict the presence of HMRs box-like sequences within the above target promoter sequences by using the matrixscan tool ${ }^{1}$. The higher weighted score for the target gene represented the higher probability of direct protein and DNA sequence association. With the weight score cut-off of 6.0 , the HMRs box-like sequences were predicted for $t c p P$ and $t c p A$, but not for toxR and toxT (Table 2), suggesting the possible direct binding of HapR on the promoter regions of these two genes.

We considered that Fur may also possibly to regulate the expression or assembly of TCP, since the fur mutant exhibited reduced TCP expression and weak autoagglutination (Mey et al., 2005). In our study, the Fur binding box (Mey et al., 2005; Davies et al., 2011) was used as well for prediction of Fur box-like sequences within the promoter regions of toxR, $t c p P$, tox $T$, and tcpA. Fur box-like sequences were predicted from each promoter of toxT, $t c p P$, and $t c p A$, but none was detected for the toxR promoter (Table 2). Expression of Fur itself was directly and negatively regulated by HapR in V. cholerae (Gao et al., 2018), thus, we speculated that the transcription of $t c p P$, toxT, and $t c p A$ might be regulated under the collective effects of HapR and Fur in a subtle manner in $V$. cholerae.

\section{Transcription of hapR, fur, tcpP, toxT, and tcpA Were Cell Density-Dependent in the TCP-Induced AKI Culture Condition}

A transcriptional luminescence reporter assay was applied to detect the transcriptional changes of hapR, fur, toxR, tcp $P$, toxT, and $t c p A$ during the growth periods of $V$. cholerae in the TCP-induced AKI culture condition. As shown in Figure 2, the transcriptional patterns of all of the genes tested were manifested in a cell-density dependent manner. The highest transcriptional levels of hapR and fur occurred at an $\mathrm{OD}_{600}$ value of around 1.0 and 0.7 , respectively, which were consistent with that described in a previous report (Gao et al., 2018). In addition, the transcriptional levels of $t c p P / t c p A$ and toxR/toxT obviously increased with the increase of cell density from 0 to 0.4 and from 0 to 0.6 , respectively, and then declined with the further increase of the cell density. The bacteria cells were harvested at

\footnotetext{
${ }^{1}$ http://embnet.ccg.unam.mx/rsat/
} 
TABLE 2 | Predicted HMRs/Fur box-like sequences within target promoters.

\begin{tabular}{|c|c|c|c|c|c|c|c|}
\hline \multirow[b]{2}{*}{ Operon } & \multirow[b]{2}{*}{ First gene } & \multicolumn{3}{|c|}{ Fur box-like sequence } & \multicolumn{3}{|c|}{ HMRs box-like sequence } \\
\hline & & Position \& & Sequence & Score & Position\& & Sequence & Score \\
\hline \multirow{2}{*}{ toxRS } & toxR & NA & NA & NA & NA & NA & NA \\
\hline & toxT & D-611...-593 & AATGAAATITATCCTCATA & 8.9 & NA & NA & NA \\
\hline tcpPH & tcpP & $D-459 \ldots-441$ & AATTATाПППTATCATT & 9.4 & $R-71 \ldots-52$ & ППTAATATAATTATाTGCA & 7.7 \\
\hline tcpA-F & tcpA & $D-273 \ldots-255$ & AACGCATITATTTGCATT & 7.0 & $D-125 \ldots-106$ & AAAAATGATATCTGTCAATT & 6.1 \\
\hline
\end{tabular}

\& ' $D$ ' indicates the direct sequence while ' $R$ ' the reverse one; minus numbers denote the nucleotide positions upstream of indicated genes; 'NA' represents 'not applicable.'

the $\mathrm{OD}_{600}$ value of about 1.0 and 0.7 for characterizing HapRand Fur-mediated gene regulation, respectively.

\section{HapR Repressed Transcription of tcpP and tcpA Directly, and Repressed toxT Indirectly}

The qPCR results showed that the mRNA transcription of $t c p P$, toxT, and $t c p A$ were greatly increased in $\Delta h a p R$ relative to WT (Figure 3A), while that of toxR manifested no obvious difference between $\triangle h a p R$ and WT (Supplementary Figure S1A). In addition, the luminescence assays showed that the promoter activities of $t c p P$, toxT, and $t c p A$ in $\Delta h a p R$ were much higher than that in WT (Figure 3B), whereas that under the control of toxR promoter showed a similar magnitude in $\triangle h a p R$ and WT (Supplementary Figure S1B). The DNase I footprinting assay disclosed that His-HapR protected a single DNA region within each of the promoters of $t c p P$ and $t c p A$, located from 48 to 14 and 127 to 95 upstream of $t c p P$ and $t c p A$ against DNase I digestion (Figure 3C), but no HapR binding sites were detected for toxT and toxR (Figure 3C and Supplementary Figure S1C). Thus, HapR inhibits the transcription of $\operatorname{tcp} P$ and $t c p A$ in a

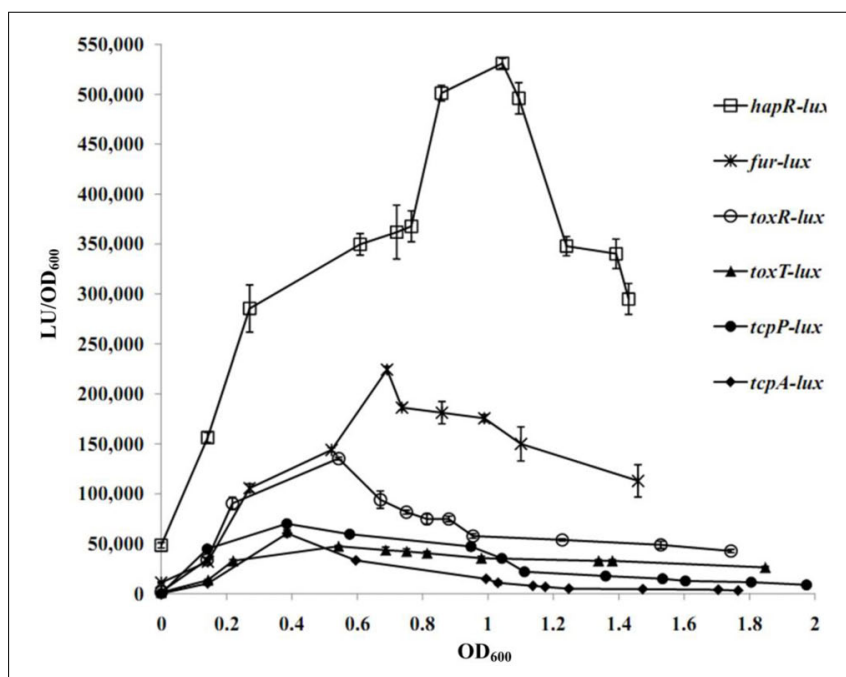

FIGURE 2 | Cell density-dependent expression of target genes. The WT strain was transformed with a recombinant pBBRlux vector that contains a promoter DNA region of the target gene. The bacteria were cultivated in the TCP-induced AKI conditions to determine the luminescence activity under various $\mathrm{OD}_{600}$ values direct manner, but it indirectly represses tox $T$ transcription and manifests no regulatory action on tox $R$ transcription.

\section{Fur Directly Activates the Transcriptions of $\operatorname{tcp} P$, toxT, and $\operatorname{tcp} A$}

The qPCR assay was employed to investigate the regulatory effects of Fur on the transcription of toxR, tcpP, toxT, and $t c p A$, and the results showed that the mRNA levels of $t c p P$, toxT, and $t c p A$ were obviously decreased in $\Delta$ fur relative to WT (Figure 4A), while that of toxR manifested no obvious difference between $\Delta$ fur and WT (Supplementary Figure S2A). These results suggested that Fur activates the transcription of $t c p P$, toxT, and $\operatorname{tcp} A$, but it seems to have no regulatory activity on toxR transcription. In addition, the promoter DNA region of toxR, $t c p P$, tox $T$, and $t c p A$ was each cloned into the pBBRlux plasmid, and then transferred into $\Delta$ fur and WT, respectively, to test the regulatory actions of Fur on their promoter activities. The results showed that the luminescence under the control of $t c p P$, toxT, or $t c p A$ promoter in $\Delta f u r$ was much lower than that in WT (Figure 4B), whereas that under the control of toxR promoter showed a similar magnitude in $\Delta$ fur and WT (Supplementary Figure S2B). As further determined by the DNase I footprinting assay, His-Fur protected a single DNA region within each of the promoters of $t c p P$, toxT, and $t c p A$, located from 524 to 446,626 to 537 , and 282 to 198 upstream of $t c p P$, toxT, and $t c p A$ against DNase I digestion in a dose-dependent manner (Figure 4C), but no binding sites were detected for toxR (Supplementary Figure S2C). Thus, Fur activates the transcription of $t c p P$, toxT, and tcpA in a direct manner, but has no regulatory activity on tox $R$ transcription.

\section{Identification of the Transcription Start Site for tcpA}

The 500 bp upstream DNA regions of $t c p P$, toxT, and tcpA in El Tor strain C7258 share a high identity (90, 99, and 87\%, respectively) in nucleotide sequences with that of in classical biotype strain O395, in which the transcription start sites of these genes have been previously reported (Higgins and DiRita, 1994; Kovacikova and Skorupski, 2001; Goss et al., 2010; Stonehouse et al., 2011) (also seen in Supplementary Figure S3). However, it was notable that the nucleotides in the position of the transcription start site of $t c p A$ are different in these two strains (Supplementary Figure S3). Thus, in this study, we mapped the transcription start site of $t c p A$ using the primer extension assay. As shown in Supplementary Figure S4, the assay detected only 


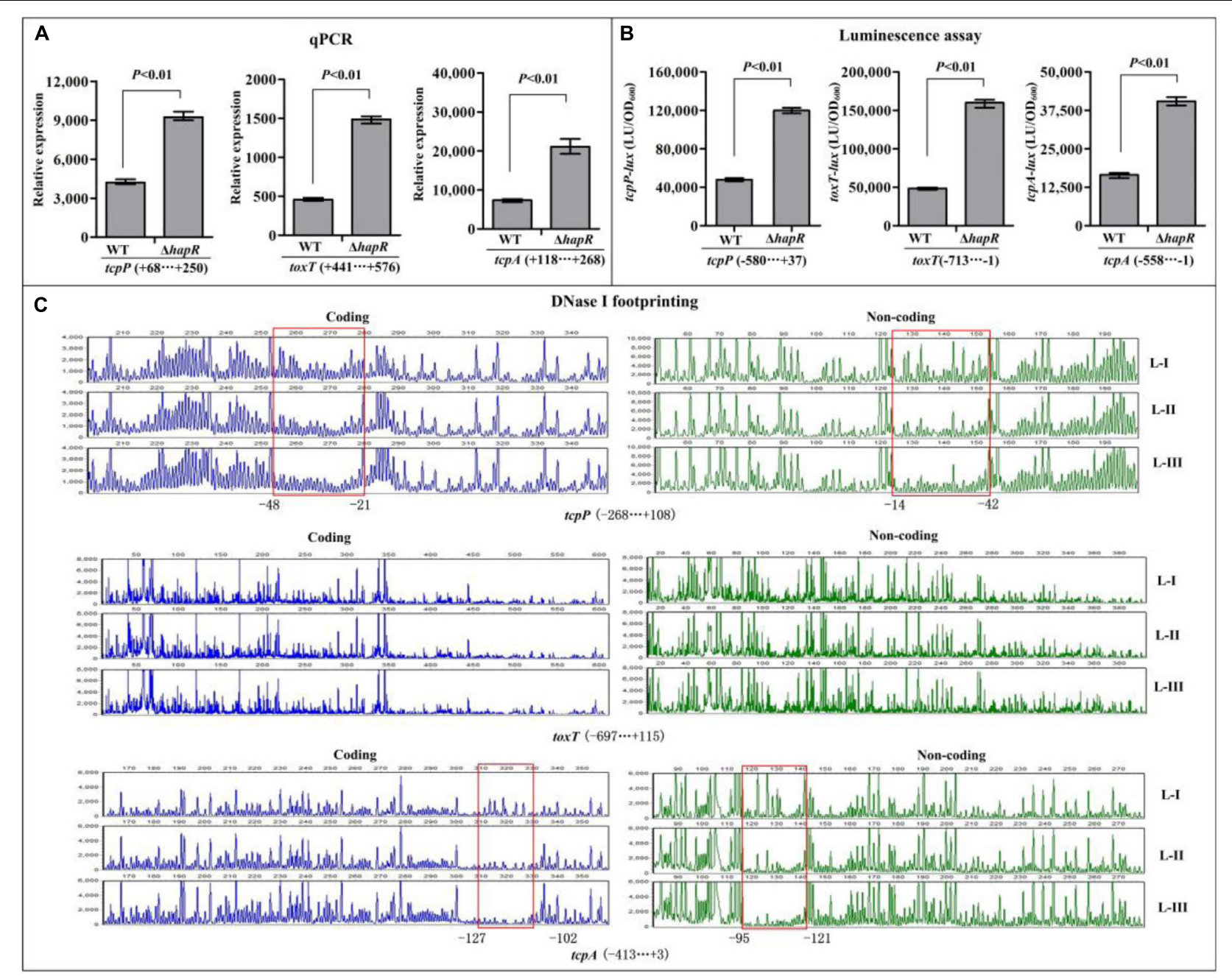

FIGURE 3 | Regulation of tcpP, toxT, and tcpA by HapR. (A) qPCR. Relative mRNA levels of the target genes were compared between $\triangle$ hapR and WT. (B) The luminescence assay was done as shown in Figure 2. (C) DNase I footprinting. The promoter DNA fragment of each target gene was labeled with FAM and HEX, respectively, and incubated with increasing amounts of purified His-HapR (L-I, -II, and - III contain 0, 2.31, and 6.92 pmol, respectively). After being digested with DNase I, the fragments were analyzed using an ABI 3500XL DNA analyzer. The protected regions are boxed and marked with positions. The negative and positive numbers indicate the nucleotide positions relative to the translation start site $(+1)$ of the corresponding gene.

one transcription start site for $t c p A$ located at $74 \mathrm{bp}$ upstream of the coding region, the position of which is exactly the same as that of in O395, suggesting that a point mutation in this position has occurred in the two strains. The -10 element is good match with the consensus prokaryotic sequence, but the -35 element is non-conservative, suggesting that $t c p A$ possess a relatively weak promoter (Figure 6).

\section{Fur but Not HapR Plays a Role in Intestinal Colonization of $V$. cholerae in Infant Mice}

The in vivo competition assay was employed to further investigate the ability of $\Delta$ fur and $\triangle h a p R$ strains to colonize the small intestine of infant mice in comparison with the $\Delta$ lacZ strain (Figure 5). The results showed that the colonization ability of $\Delta$ fur was attenuated approximately 10 -fold, while that of
$\Delta h a p R$ seemed to have no obvious difference compared to $\Delta$ lacZ $(\mathrm{CI} \approx 1)$. The same extent colonization capacity of $\Delta h a p R$ as wild-type $V$. cholerae has been previously reported (Zhu et al., 2002; Zhu and Mekalanos, 2003). These results suggested that Fur but not HapR is required for efficient colonization.

\section{DISCUSSION}

Vibrio cholerae expresses the virulence determinants to establish colonization in the gut and cause disease diarrhea. Expression of TCP and CT is highly regulated by environmental stimuli and a variety of regulators (Taylor et al., 1987; Miller and Mekalanos, 1988; Nye et al., 2000; Kovacikova and Skorupski, 2001; Zhu et al., 2002; Kovacikova et al., 2004; Mey et al., 2005; Childers and Klose, 2007). The regulatory cascade, which is constituted of ToxR, TcpP, and ToxT referred to as the ToxR virulence 


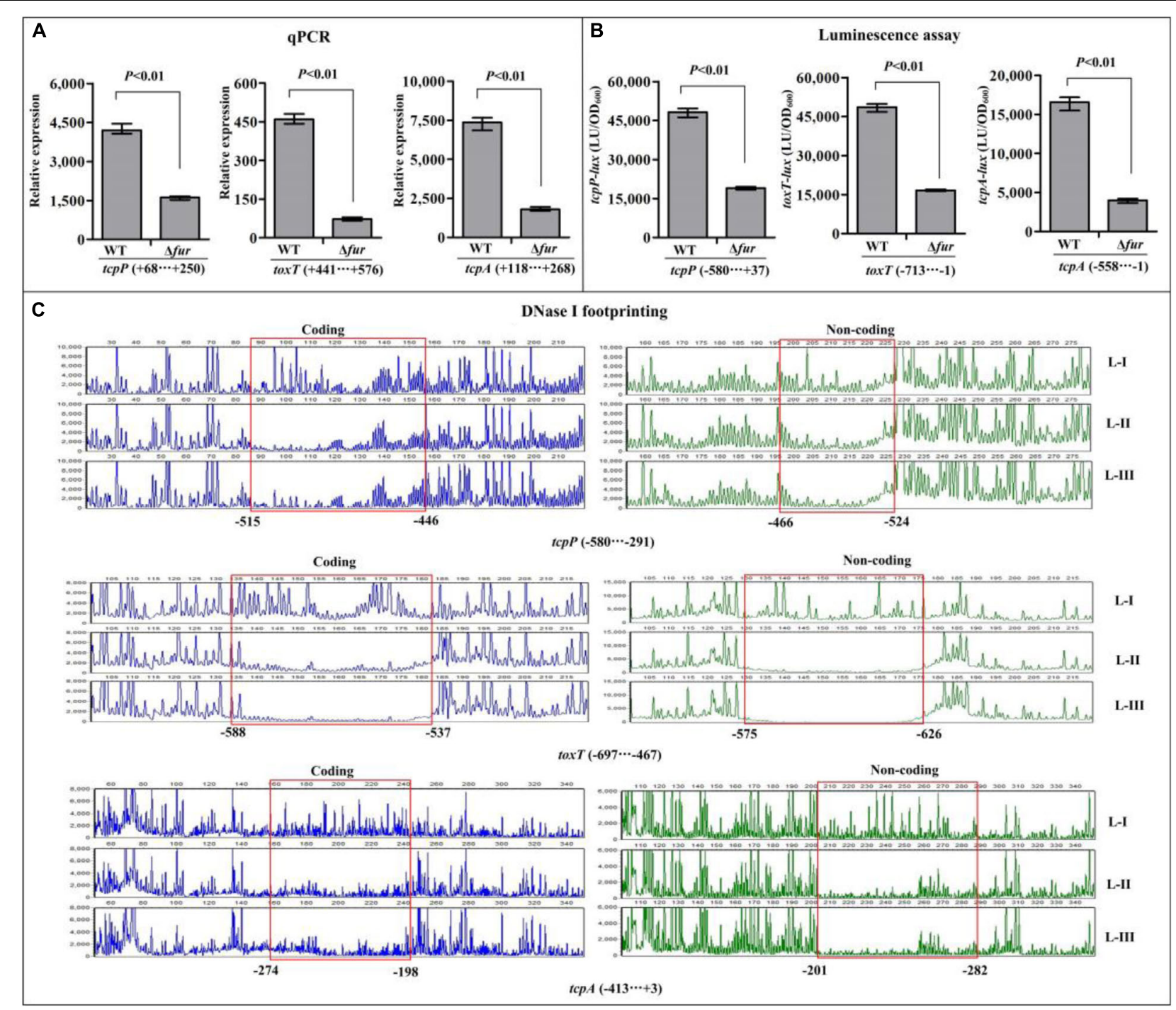

FIGURE 4 | Regulation of tcpP, toxT, and tcpA by Fur. The qPCR (A) and DNase I footprinting assays (C) were done as Figure $\mathbf{3}$, while the luminescence assay (B) was done as Figure 2. L-I, -II, and -III contain 0, 2.95, and 8.85 pmol of His-Fur, respectively.

regulon, has been shown to be involved in the regulation of TCP and CT, and the regulatory mechanisms also have been well documented (DiRita et al., 1991; Krukonis et al., 2000; Goss et al., 2013). Other regulatory factors modulate TCP and CT production mostly via regulation of the genes within this cascade (Kovacikova and Skorupski, 2001; Kovacikova et al., 2004). HapR also has been shown to be involved in regulating the transcription of $t c p P$, toxT, and $t c p A$ (Kovacikova and Skorupski, 2002; Zhu et al., 2002), but lacks the direct evidence for its binding to their regulatory regions.

In the present study, we found a HMRs box-like sequence for each promoter of $t c p P$ and $t c p A$, suggesting that the transcription of $t c p P$ and $t c p A$ would be under the direct control of HapR in $V$. cholerae. We observed that HapR binds to the promoters of tcpP and tcpA to repress their transcription when the bacterial cells were harvested at an $\mathrm{OD}_{600}$ value of about 1.0. The HapR binding site for each $t c p P$ and $t c p A$ promoter overlaps the core -10 and/or -35 elements, and thus HapR repression of $t c p P$ and $t c p A$ transcription would be via blocking the entry or elongation of the RNA polymerase. In addition, we noticed that the HapR binding site for $t c p A$ promoter partly overlaps with the sequence protected by ToxT (Yu and DiRita, 2002; Withey and DiRita, 2006), which acts as an activator of tcpA. Thus, the binding of HapR to the tcpA promoter may prevent ToxT from binding to. However, the binding site of HapR for $t c p P$ promoter excludes the HMRs box-like sequence, suggesting the results of the informatics analysis are not always reliable. HapR also appears to repress the transcription of tox $T$ in an indirect manner, which differs from its interaction with $t c p P$ and $t c p A$ promoters and could involve additional regulators. A previously study showed that ToxR and TcpP bind adjacently to the promoter DNA region of toxT (Figure 6), and the interaction of ToxR and TcpP with 


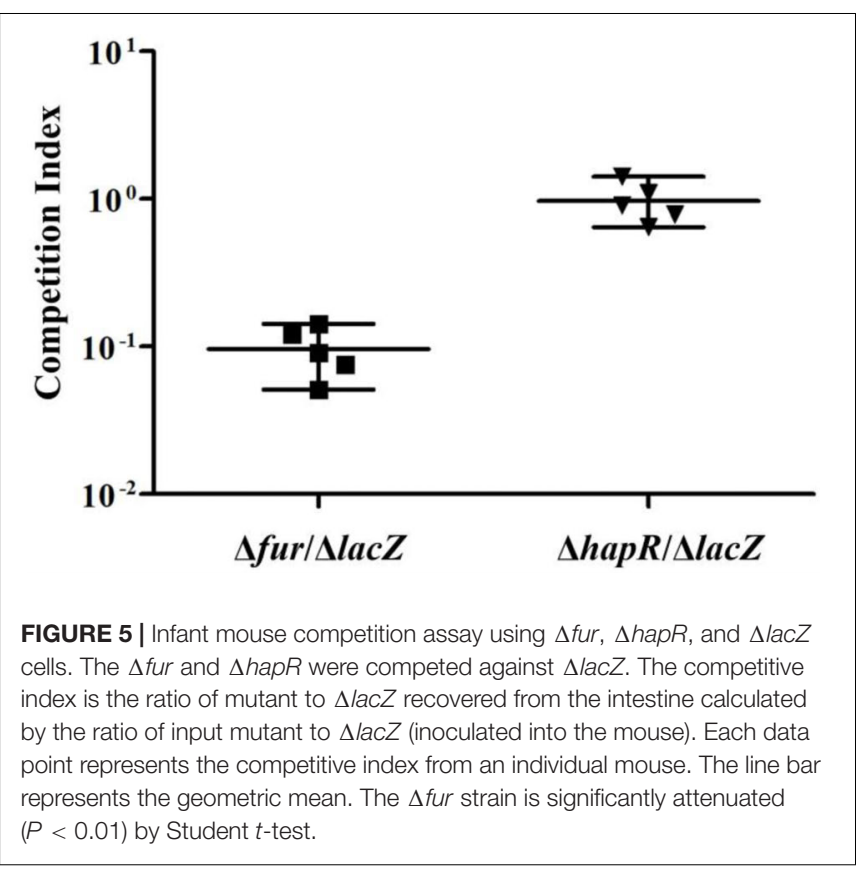

the toxT promoter enhanced the ability of TcpP to interact with RNA polymerase, leading to transcriptional activation of toxT (Krukonis et al., 2000). Thus, HapR repression of toxT promoter may be via the directly repression of $t c p P$ transcription by HapR.

HapR is a global regulator that controls the expression of hundreds of genes, particularly those responsible for the motility, biofilm formation, metabolism, and virulence (Ball et al., 2017). HapR regulon contains a variety of transcriptional regulator genes, such as aphA (Kovacikova and Skorupski, 2002), vpsT (Waters et al., 2008), qstR (Lo Scrudato and Blokesch, 2013), and fur (Gao et al., 2018). The regulatory actions of HapR on other regulatory genes bridge the different regulons in $V$. cholerae, forming complex regulatory networks to tightly control gene expression. For instance, HapR directly regulated the expression of Fur and HlyU, and these regulators coordinately regulated the transcription of $h l y A$, leading to the highest expression level of $h l y A$ occurring at the early mid-logarithmic growth phase (Gao et al., 2018). Although the regulatory mechanisms are still obscure, Fur-dependent TCP production has been observed in $V$. cholerae (Mey et al., 2005). Thus, we deduced that expression of TCP is also coordinately regulated by HapR and Fur in V. cholerae.

The binding sites of Fur usually contain a 19 bp inverted repeat sequence known as the classic Fur box (Escolar et al., 1999; Mey et al., 2005; Zhou et al., 2006). A 21 bp palindromic sequence was also reported as the enhanced Fur box (Davies et al., 2011), but it shares sequence similarity with the previously predicted (Mey et al., 2005). The Fur box-like sequences were detected for the promoters of tox $T, t c p P$, and $t c p A$. Indeed, the transcription of the three above genes was under the direct and positive control of Fur at $\mathrm{OD}_{600}$ value of about 0.7 . The Fur binding site for each target promoter located far upstream of the core -35 element. Thus, Fur activation of toxT, tcp $P$, and $t c p A$ transcription may belong to the class I stimulation mechanism (Ishihama, 2000). However, Fur binding sites for toxT, $t c p P$, and $t c p A$ were not identified by ChIP-seq analysis (Davies et al., 2011). Discrepancies between the present data and the data identified by ChIP-seq could be attributed to the different experimental methods, growth conditions and/or bacterial genetic background, because the ChIP-seq is used to map the binding sites of a regulator in vivo. Anyhow, the data presented here showed that Fur activation of TCP production is via at least direct activation of toxT and $t c p P$ transcription, as well as the TCP coding gene tcpA.

Toxin coregulated pilus mostly contributes to the colonization of $V$. cholerae in the host intestine (Kirn et al., 2000). We thus investigated the colonization ability of fur and hapR deletion mutants in the small intestine of an infant mouse model. The results showed that the colonization ability of $\Delta$ fur was significantly attenuated relative to that of the $\Delta$ lac $Z$ strain, which was consistent with previous report (Mey et al., 2005). Fur is an iron-dependent transcriptional regulator that regulates the expression of multitudinous genes involved in iron homeostasis, virulence, biofilm formation, ribosome formation, transporters, porin proteins, and unique sRNAs (Occhino et al., 1998; Davis et al., 2005; Mey et al., 2005; Wyckoff et al., 2007; Craig et al., 2011; Davies et al., 2011; Sun et al., 2012a). Iron is an essential metallic element for life, but there is almost no free iron to use within the intestine of humans at the early infection stage of $V$. cholerae (Ganz, 2018). It was reported that the stools from cholera patients contain a heterogenous mixture of biofilm-like aggregates, and the biofilm formation is initiated almost immediately after adherence of $V$. cholerae to intestinal cells (Faruque et al., 2006; Sengupta et al., 2016). Our unpublished data showed that $\Delta$ fur produces more biofilms than the WT strain under the iron-starved growth condition. The attenuated colonization ability of $\Delta$ fur could be attributed to the significantly enhanced biofilm formation and the low production of TCP. The ability of colonization of $\Delta h a p R$ showed no obvious difference compared to that of the $\Delta$ lac $Z$ strain, the same as previously reported (Zhu et al., 2002). However, the $\Delta$ hapR strain showed a 10 -fold colonization defect relative to the WT strain, when used as the inoculum composed of biofilms to infect infant mice (Zhu and Mekalanos, 2003). The $\Delta$ hapR strains formed much thicker biofilms, but the detachment of $\Delta h a p R$ from biofilms was defective, which may influence the HapRdependent colonization in biofilms (Zhu and Mekalanos, 2003). In a word, Fur-dependent but HapR-independent colonization in planktonic cells would be beneficial to the pathogenesis and transmission of $V$. cholerae during the disease progression.

The highest expression levels of $t c p P$, tox $T$, and tcpA occurred at an $\mathrm{OD}_{600}$ value of around 0.4 , but the lower expression levels were observed at both LCD and HCD. AphA is the bottom master regulator of QS that operates at LCD (Lu et al., 2018). However, a previous study showed that AphA binds to the promoter of tcpPH to activate its transcription (Kovacikova et al., 2004). TcpP, in turn, activates toxT and tcpA transcription (DiRita et al., 1991; Krukonis et al., 2000). Thus, we deduced that: at LCD or at the initial stage of infection, AphA activates the expression of TCP and CT via direct activation of tcpP expression, which promotes colonization and induces watery diarrhea. However, 
$\operatorname{tox} T$

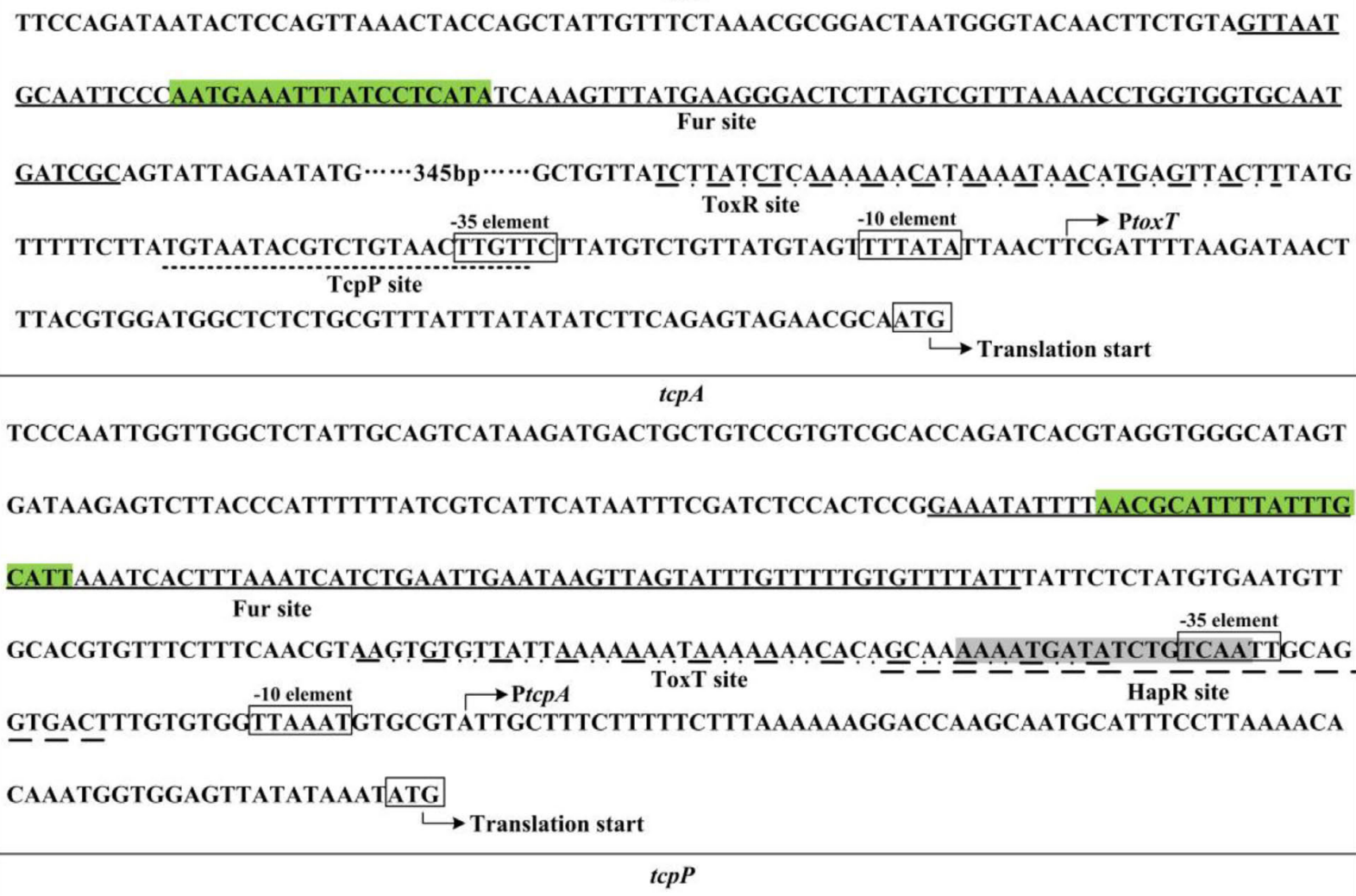

GTGCCTGCTGAGAACTAAGGCTAGATATAGATTTTGTTTGATCTGATAGTTTCGAGTGATAGAAAAAGGCAGTAAAA GCCAACGTAATGATGCACGCAAGTAATAAAAAAACCGAAATTATTTTTTTTATCATTAGTTTAACTCTAAGTTTAAAT Fur site

GGTTATCACGGAGTACTTCGTGATAATTAGTTAAAAATGAAATTATAAAATAATGATGTGAAAAATCAGCTTTTATCG TTTTAAATAGTGTTTTTGTCTTTAGGAAAATAAAGTTACAAAGTTAAAAAAAGCCCCAAACGTAAGGGGCAAAATGTC ACAGGAAAGATAATGTAACCAAGTTAATAGATATGGAATAGGCACTATAGGGgGGAGTGCTAAACCGAATGAATTAT AATGAGAATTACTTTCTGATATCGTGACTGGTTATTTTTTTAATGCTGTTTTATTTTTTTTGAATGGCTTGGTTATTAT GCAATTAAGTTCTCATTATCAACTGCAGAATTAGATTGCAAATAATTATATTAAAAAAAA TAGACA

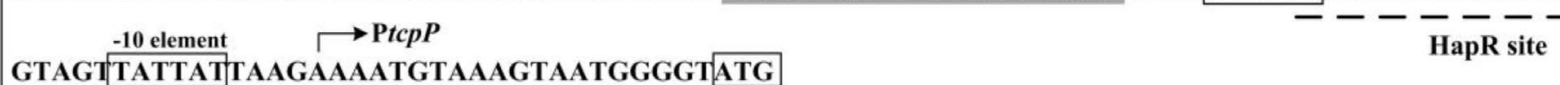

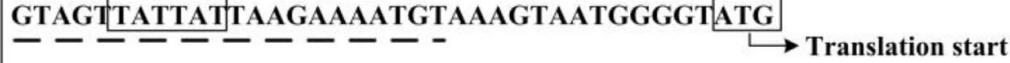

Fur box-like sequences

HapR box-like sequences

FIGURE 6 | Promoter organization of target genes. The sequences were derived from Vibrio cholerae EI Tor C7258. The transcription/translation start sites are indicated by bent arrows. The -10 and -35 elements are enclosed in boxes. The binding sites of ToxR and TcpP identified by DNase I digestion were previously reported by Krukonis et al. (2000). The sequence protected by ToxT in the promoter DNA region of tcpA was identified by Yu and DiRita (2002) and Withey and DiRita (2006). The Fur sites are underlined with solid lines, whereas the HapR sites are underlined with broken lines.

the intracellular level of AphA protein is rapidly degraded with the increase of cell density (Rutherford et al., 2011). At this point, Fur may be engaged to further activate the expression of TCP through binding and activation of the transcription of $t c p P$, toxT, and $t c p A$. While at HCD or at the later stage of infection (high number of $V$. cholerae cells in the intestine), high expressed HapR binds to the promoters of $t c p P$ and $t c p A$ to repress their transcription, and thus inhibits TCP and CT 
production, which then enables the pathogen to detach from the epithelium, and exits the host along with the stool. In addition, the transcriptional pattern of tox $R$ also manifested a cell-density dependent manner, with a similar results previously observed in $V$. cholerae and the closely related Vibrio parahaemolyticus (Xu et al., 2010; Zhang et al., 2017a). However, both HapR and Fur seemed to have no regulatory actions on tox $R$ transcription under the current growth conditions. The molecular mechanism of celldensity dependent expression of toxR, therefore, needs to be further investigated. Nevertheless, the present work enriched the virulence regulatory networks in $V$. cholerae, and broadens our understanding of the pathogenic mechanisms of the pathogen.

\section{DATA AVAILABILITY STATEMENT}

The raw data supporting the conclusions of this article will be made available by the authors, without undue reservation, to any qualified researcher.

\section{ETHICS STATEMENT}

The animal study was reviewed and approved by the ethics committee of the National Institute for Communicable Disease Control and Prevention, China CDC. Written informed consent was obtained from the owners for the participation of their animals in this study.

\section{AUTHOR CONTRIBUTIONS}

HG, YZ, and BK conceived the study and designed experimental procedures. JZ, JLo, JLi, QQ, and QS performed the experiments and carried out data analysis. $\mathrm{HG}, \mathrm{YZ}$, and $\mathrm{BK}$ wrote the manuscript.

\section{REFERENCES}

Abuaita, B. H., and Withey, J. H. (2011). Termination of Vibrio cholerae virulence gene expression is mediated by proteolysis of the major virulence activator, ToxT. Mol. Microbiol. 81, 1640-1653. doi: 10.1111/j.1365-2958.2011.07798.x

Ball, A. S., Chaparian, R. R., and van Kessel, J. C. (2017). Quorum sensing gene regulation by LuxR/HapR master regulators in Vibrios. J. Bacteriol. 199, e105-e117. doi: 10.1128/JB.00105-17

Childers, B. M., and Klose, K. E. (2007). Regulation of virulence in Vibrio cholerae: the ToxR regulon. Future Microbiol. 2, 335-344. doi: 10.2217/17460913.2.3.335

Clemens, J. D., Nair, G. B., Ahmed, T., Qadri, F., and Holmgren, J. (2017). Cholera. Lancet 390, 1539-1549. doi: 10.1016/S0140-6736(17)30559-7

Craig, S. A., Carpenter, C. D., Mey, A. R., Wyckoff, E. E., and Payne, S. M. (2011). Positive regulation of the Vibrio cholerae porin OmpT by iron and fur. J. Bacteriol. 193, 6505-6511. doi: 10.1128/JB.05681-11

Davies, B. W., Bogard, R. W., and Mekalanos, J. J. (2011). Mapping the regulon of Vibrio cholerae ferric uptake regulator expands its known network of gene regulation. Proc. Natl. Acad. Sci. U.S.A. 108, 12467-12472. doi: 10.1073/pnas. 1107894108

Davis, B. M., Quinones, M., Pratt, J., Ding, Y., and Waldor, M. K. (2005). Characterization of the small untranslated RNA RyhB and its regulon in Vibrio cholerae. J. Bacteriol. 187, 4005-4014. doi: 10.1128/JB.187.12.4005-4014.2005

\section{FUNDING}

This study was supported by grants from the National Natural Science Foundation of China (Grant No. 81471917) and the Science Foundation for the State Key Laboratory for Infectious Disease Prevention and Control from China (Grant No. 2015SKLID509).

\section{ACKNOWLEDGMENTS}

We thank Dr. George Osei-Adjei from the Republic of Ghana for language editing the manuscript.

\section{SUPPLEMENTARY MATERIAL}

The Supplementary Material for this article can be found online at: https://www.frontiersin.org/articles/10.3389/fmicb. 2020.00709/full\#supplementary-material

FIGURE S1 | Regulation of toxR by HapR. The qPCR (A) and DNase I footprinting assays were done as in Figure 3, while the luminescence assay (B) was done as in Figure 2. L-I, -II, and -III contain 0, 2.31, and 6.92 pmol of His-HapR, respectively.

FIGURE S2 | Regulation of toxR by Fur. The qPCR (A) and DNase I footprinting assays were done as Figure $\mathbf{3}$, while the luminescence assay (B) was done as in Figure 2. L-I, -II, and -III contain 0, 2.95, and 8.85 pmol of His-Fur, respectively.

FIGURE S3 | The nucleotide sequences alignment of the promoter DNA regions of tcpP and toxT. The promoter DNA sequences of tcpP, toxT and tcpA were derived from $V$. cholerae El Tor biotype strain C7258 and classical biotype strain 0395. The different bases were labeledred, while the identical bases were marked with asterisk $\left({ }^{*}\right)$. Shown also were the transcription start sites, -10 and -35 boxes.

FIGURE S4 | Transcription start site of tcpA in V. cholerae El Tor biotype strain C7258. A 5'-HEX-labeled reverse primer was designed to be complementary to the RNA transcript of $t c p A$. The primer extension products were analyzed with an ABI 3500XL DNA Genetic analyzer. The transcription start site was marked with asterisks andpositions.

DiRita, V. J., Parsot, C., Jander, G., and Mekalanos, J. J. (1991). Regulatory cascade controls virulence in Vibrio cholerae. Proc. Natl. Acad. Sci. U.S.A. 88, 5403-5407. doi: $10.1073 /$ pnas.88.12.5403

Escolar, L., Perez-Martin, J., and de Lorenzo, V. (1999). Opening the iron box: transcriptional metalloregulation by the Fur protein. J. Bacteriol. 181, 62236229. doi: 10.1128/jb.181.20.6223-6229.1999

Faruque, S. M., Biswas, K., Udden, S. M., Ahmad, Q. S., Sack, D. A., Nair, G. B., et al. (2006). Transmissibility of cholera: in vivo-formed biofilms and their relationship to infectivity and persistence in the environment. Proc. Natl. Acad. Sci. U.S.A. 103, 6350-6355. doi: 10.1073/pnas.0601277103

Ganz, T. (2018). Iron and infection. Int. J. Hematol. 107, 7-15. doi: 10.1007/s12185017-2366-2

Gao, H., Xu, J., Lu, X., Li, J., Lou, J., Zhao, H., et al. (2018). Expression of hemolysin is regulated under the collective actions of HapR, Fur, and HlyU in Vibrio cholerae El Tor serogroup O1. Front. Microbiol. 9:1310. doi: 10.3389/fmicb. 2018.01310

Gao, H., Zhang, L., Osei-Adjei, G., Yang, W., Zhou, D., Huang, X., et al. (2017). Transcriptional regulation of Q-mfpABC and mfpABC by CalR in Vibrio parahaemolyticus. Microbiologyopen 6:e00470. doi: 10.1002/mbo3.470

Gao, H., Zhang, Y., Yang, L., Liu, X., Guo, Z., Tan, Y., et al. (2011). Regulatory effects of cAMP receptor protein (CRP) on porin genes and its own gene in Yersinia pestis. BMC Microbiol. 11:40. doi: 10.1186/1471-2180-11-40 
Gao, H., Zhou, D., Li, Y., Guo, Z., Han, Y., Song, Y., et al. (2008). The ironresponsive Fur regulon in Yersinia pestis. J. Bacteriol. 190, 3063-3075. doi: 10.1128/JB.01910-07

Goss, T. J., Morgan, S. J., French, E. L., and Krukonis, E. S. (2013). ToxR recognizes a direct repeat element in the toxT, ompU, ompT, and ctxA promoters of Vibrio cholerae to regulate transcription. Infect. Immun. 81, 884-895. doi: 10.1128/IAI. 00889-12

Goss, T. J., Seaborn, C. P., Gray, M. D., and Krukonis, E. S. (2010). Identification of the TcpP-binding site in the toxT promoter of Vibrio cholerae and the role of ToxR in TcpP-mediated activation. Infect. Immun. 78, 4122-4133. doi: 10.1128/ IAI.00566- 10

Henke, J. M., and Bassler, B. L. (2004). Three parallel quorum-sensing systems regulate gene expression in Vibrio harveyi. J. Bacteriol. 186, 6902-6914. doi: 10.1128/JB.186.20.6902-6914.2004

Higgins, D. E., and DiRita, V. J. (1994). Transcriptional control of toxT, a regulatory gene in the ToxR regulon of Vibrio cholerae. Mol. Microbiol. 14, 17-29. doi: 10.1111/j.1365-2958.1994.tb01263.x

Hung, D. T., and Mekalanos, J. J. (2005). Bile acids induce cholera toxin expression in Vibrio cholerae in a ToxT-independent manner. Proc. Natl. Acad. Sci. U.S.A. 102, 3028-3033. doi: 10.1073/pnas.0409559102

Ishihama, A. (2000). Functional modulation of Escherichia coli RNA polymerase. Annu. Rev. Microbiol. 54, 499-518. doi: 10.1146/annurev.micro.54.1.499

Iwanaga, M., Yamamoto, K., Higa, N., Ichinose, Y., Nakasone, N., and Tanabe, M. (1986). Culture conditions for stimulating cholera toxin production by Vibrio cholerae O1 El Tor. Microbiol. Immunol. 30, 1075-1083. doi: 10.1111/j.13480421.1986.tb03037.x

Kirn, T. J., Lafferty, M. J., Sandoe, C. M., and Taylor, R. K. (2000). Delineation of pilin domains required for bacterial association into microcolonies and intestinal colonization by Vibrio cholerae. Mol. Microbiol. 35, 896-910. doi: 10.1046/j.1365-2958.2000.01764.x

Kleber-Janke, T., and Becker, W. M. (2000). Use of modified BL21(DE3) Escherichia coli cells for high-level expression of recombinant peanut allergens affected by poor codon usage. Protein Express. Purif. 19, 419-424. doi: 10.1006/prep.2000. 1265

Kovacikova, G., Lin, W., and Skorupski, K. (2004). Vibrio cholerae AphA uses a novel mechanism for virulence gene activation that involves interaction with the LysR-type regulator AphB at the tcpPH promoter. Mol. Microbiol. 53, 129-142. doi: 10.1111/j.1365-2958.2004.04121.x

Kovacikova, G., and Skorupski, K. (2001). Overlapping binding sites for the virulence gene regulators $\mathrm{AphA}, \mathrm{AphB}$ and cAMP-CRP at the Vibrio cholerae tcpPH promoter. Mol. Microbiol. 41, 393-407. doi: 10.1046/j.1365-2958.2001. 02518.x

Kovacikova, G., and Skorupski, K. (2002). Regulation of virulence gene expression in Vibrio cholerae by quorum sensing: HapR functions at the aphA promoter. Mol. Microbiol. 46, 1135-1147. doi: 10.1046/j.1365-2958.2002. 03229.x

Krukonis, E. S., Yu, R. R., and Dirita, V. J. (2000). The Vibrio cholerae ToxR/TcpP/ToxT virulence cascade: distinct roles for two membrane-localized transcriptional activators on a single promoter. Mol. Microbiol. 38, 67-84. doi: 10.1046/j.1365-2958.2000.02111.x

Lee, H. J., Bang, S. H., Lee, K. H., and Park, S. J. (2007). Positive regulation of fur gene expression via direct interaction of fur in a pathogenic bacterium, Vibrio vulnificus. J. Bacteriol. 189, 2629-2636. doi: 10.1128/JB.01791-06

Lo Scrudato, M., and Blokesch, M. (2013). A transcriptional regulator linking quorum sensing and chitin induction to render Vibrio cholerae naturally transformable. Nucleic Acids Res. 41, 3644-3658. doi: 10.1093/nar/gkt041

Lu, R., Osei-Adjei, G., Huang, X., and Zhang, Y. (2018). Role and regulation of the orphan AphA protein of quorum sensing in pathogenic Vibrios. Future Microbiol. 13, 383-391. doi: 10.2217/fmb-2017-0165

Matson, J. S., and DiRita, V. J. (2005). Degradation of the membrane-localized virulence activator TcpP by the YaeL protease in Vibrio cholerae. Proc. Natl. Acad. Sci. U.S.A. 102, 16403-16408. doi: 10.1073/pnas.0505818102

Merritt, E. A., and Hol, W. G. (1995). AB5 toxins. Curr. Opin. Struct Biolo. 5, 165-171. doi: 10.1016/0959-440x(95)80071-9

Mey, A. R., Wyckoff, E. E., Kanukurthy, V., Fisher, C. R., and Payne, S. M. (2005). Iron and fur regulation in Vibrio cholerae and the role of fur in virulence. Infect. Immun. 73, 8167-8178. doi: 10.1128/IAI.73.12.8167-8178.2005
Miller, M. B., Skorupski, K., Lenz, D. H., Taylor, R. K., and Bassler, B. L. (2002). Parallel quorum sensing systems converge to regulate virulence in Vibrio cholerae. Cell 110, 303-314. doi: 10.1016/s0092-8674(02)00829-2

Miller, V. L., and Mekalanos, J. J. (1988). A novel suicide vector and its use in construction of insertion mutations: osmoregulation of outer membrane proteins and virulence determinants in Vibrio cholerae. J. Bacteriol. 170, 2575 2583. doi: 10.1128/jb.170.6.2575-2583.1988

Nye, M. B., Pfau, J. D., Skorupski, K., and Taylor, R. K. (2000). Vibrio cholerae H-NS silences virulence gene expression at multiple steps in the ToxR regulatory cascade. J. Bacteriol. 182, 4295-4303. doi: 10.1128/jb.182.15.4295-4303. 2000

Occhino, D. A., Wyckoff, E. E., Henderson, D. P., Wrona, T. J., and Payne, S. M. (1998). Vibrio cholerae iron transport: haem transport genes are linked to one of two sets of tonB, exbB, exbD genes. Mol. Microbiol. 29, 1493-1507. doi: 10.1046/j.1365-2958.1998.01034.x

Rutherford, S. T., van Kessel, J. C., Shao, Y., and Bassler, B. L. (2011). AphA and LuxR/HapR reciprocally control quorum sensing in Vibrios. Genes Dev. 25, 397-408. doi: 10.1101/gad.2015011

Sengupta, C., Mukherjee, O., and Chowdhury, R. (2016). Adherence to Intestinal Cells Promotes Biofilm Formation in Vibrio cholerae. J. Infect. Dis. 214, 15711578. doi: 10.1093/infdis/jiw435

Stonehouse, E. A., Hulbert, R. R., Nye, M. B., Skorupski, K., and Taylor, R. K. (2011). $\mathrm{H}-\mathrm{NS}$ binding and repression of the ctx promoter in Vibrio cholerae. J. Bacteriol. 193, 979-988. doi: 10.1128/JB.01343-09

Sun, F., Gao, H., Zhang, Y., Wang, L., Fang, N., Tan, Y., et al. (2012a). Fur is a repressor of biofilm formation in Yersinia pestis. PLoS One 7:e52392. doi: 10.1371/journal.pone.0052392

Sun, F., Zhang, Y., Wang, L., Yan, X., Tan, Y., Guo, Z., et al. (2012b). Molecular characterization of direct target genes and cis-acting consensus recognized by quorum-sensing regulator AphA in Vibrio parahaemolyticus. PLoS One 7:e44210. doi: 10.1371/journal.pone.0044210

Tamayo, R., Patimalla, B., and Camilli, A. (2010). Growth in a biofilm induces a hyperinfectious phenotype in Vibrio cholerae. Infect. Immun. 78, 3560-3569. doi: 10.1128/IAI.00048-10

Taylor, R. K., Miller, V. L., Furlong, D. B., and Mekalanos, J. J. (1987). Use of phoA gene fusions to identify a pilus colonization factor coordinately regulated with cholera toxin. Proc. Natl. Acad. Sci. U.S.A. 84, 2833-2837. doi: 10.1073/pnas.84. 9.2833

Tu, K. C., Long, T., Svenningsen, S. L., Wingreen, N. S., and Bassler, B. L. (2010). Negative feedback loops involving small regulatory RNAs precisely control the Vibrio harveyi quorum-sensing response. Mol. Cell 37, 567-579. doi: 10.1016/j. molcel.2010.01.022

Waldor, M. K., and Mekalanos, J. J. (1996). Lysogenic conversion by a filamentous phage encoding cholera toxin. Science 272, 1910-1914. doi: 10.1126/science.272. 5270.1910

Waters, C. M., Lu, W., Rabinowitz, J. D., and Bassler, B. L. (2008). Quorum sensing controls biofilm formation in Vibrio cholerae through modulation of cyclic di-GMP levels and repression of vpsT. J. Bacteriol. 190, 2527-2536. doi: 10.1128/JB.01756-07

Withey, J. H., and DiRita, V. J. (2006). The toxbox: specific DNA sequence requirements for activation of Vibrio cholerae virulence genes by ToxT. Mol. Microbiol. 59, 1779-1789. doi: 10.1111/j.1365-2958.2006.05053.x

Wu, R., Zhao, M., Li, J., Gao, H., Kan, B., and Liang, W. (2015). Direct regulation of the natural competence regulator gene tfoX by cyclic AMP (cAMP) and cAMP receptor protein (CRP) in Vibrios. Sci. Rep. 5:14921. doi: 10.1038/srep 14921

Wyckoff, E. E., Mey, A. R., and Payne, S. M. (2007). Iron acquisition in Vibrio cholerae. Biometals 20, 405-416. doi: 10.1007/s10534-0069073-4

Xu, X., Stern, A. M., Liu, Z., Kan, B., and Zhu, J. (2010). Virulence regulator AphB enhances toxR transcription in Vibrio cholerae. BMC Microbiol. 10:3. doi: 10.1186/1471-2180-10-3

Yu, R. R., and DiRita, V. J. (2002). Regulation of gene expression in Vibrio cholerae by ToxT involves both antirepression and RNA polymerase stimulation. Mol. Microbiol. 43, 119-134. doi: 10.1046/j.1365-2958.2002.02721.x

Zhang, Y., Gao, H., Osei-Adjei, G., Zhang, Y., Yang, W., Yang, H., et al. (2017a). Transcriptional regulation of the type VI secretion system 1 genes by quorum 
sensing and ToxR in Vibrio parahaemolyticus. Front. Microbiol. 8:2005. doi: 10.3389/fmicb.2017.02005

Zhang, Y., Zhang, Y., Gao, H., Zhang, L., Yin, Z., Huang, X., et al. (2017b). Vibrio parahaemolyticus CalR down regulates the thermostable direct hemolysin (TDH) gene transcription and thereby inhibits hemolytic activity. Gene 613, 39-44. doi: 10.1016/j.gene.2017.03.001

Zhang, Y., Qiu, Y., Tan, Y., Guo, Z., Yang, R., and Zhou, D. (2012). Transcriptional regulation of opaR, qrr2-4 and aphA by the master quorum-sensing regulator OpaR in Vibrio parahaemolyticus. PLoS One 7:e34622. doi: 10.1371/journal. pone.0034622

Zhou, D., Qin, L., Han, Y., Qiu, J., Chen, Z., Li, B., et al. (2006). Global analysis of iron assimilation and fur regulation in Yersinia pestis. FEMS Microbiol. Lett. 258, 9-17. doi: 10.1111/j.1574-6968.2006.00208.x

Zhu, J., and Mekalanos, J. J. (2003). Quorum sensing-dependent biofilms enhance colonization in Vibrio cholerae. Dev. Cell 5, 647-656. doi: 10.1016/s15345807(03)00295-8
Zhu, J., Miller, M. B., Vance, R. E., Dziejman, M., Bassler, B. L., and Mekalanos, J. J. (2002). Quorum-sensing regulators control virulence gene expression in Vibrio cholerae. Proc. Natl. Acad. Sci. U.S.A. 99, 3129-3134. doi: 10.1073/pnas. 052694299

Conflict of Interest: The authors declare that the research was conducted in the absence of any commercial or financial relationships that could be construed as a potential conflict of interest.

Copyright (C) 2020 Gao, Zhang, Lou, Li, Qin, Shi, Zhang and Kan. This is an openaccess article distributed under the terms of the Creative Commons Attribution License (CC BY). The use, distribution or reproduction in other forums is permitted, provided the original author(s) and the copyright owner(s) are credited and that the original publication in this journal is cited, in accordance with accepted academic practice. No use, distribution or reproduction is permitted which does not comply with these terms. 